\title{
Contributos para o estudo da folha de ouro de retábulos Barrocos por microscopia óptica e electrónica
}

\author{
Ana Bidarra, João Coroado e Fernando Rocha
}

Resumo: O trabalho desenvolvido pretende fornecer uma nova perspectiva ao estudo dos retábulos Barrocos portugueses, incidindo na caracterização elementar do ouro utilizado no douramento e na identificação de elementos característicos na sua composição. Neste sentido foram analisados os retábulosmor das igrejas de São Bento da Vitória, Santa Clara e São Francisco, localizados na cidade do Porto. As análises qualitativas e semi-quantitativas foram efectuadas por microscopia óptica (OM) e microscopia electrónica de varrimento com difracção de raios-X (SEM-EDS). A análise dos resultados permitiu identificar uma liga de ouro/prata/cobre e a presença de ouro de grande pureza, entre 22 e 23 quilates.

Palavras chave: folha de ouro, retábulos barrocos, microscopia óptica, SEM-EDS

Resumen: El trabajo aquí desarrollado pretende ofrecer una nueva perspectiva para el estudio de los retablos Barrocos portugueses, incidiendo en la caracterización elemental del oro utilizado para el dorado y en la identificación de elementos característicos de su composición. En este sentido fueron analizados los retablos mayores de las iglesias de San Bento da Vitória, Santa Clara y San Francisco, localizados en la ciudad de Oporto. Los análisis cualitativos y semi cuantitativos fueron efectuados por microscopía óptica (OM) y microscopía electrónica de barrido con difracción de rayos X (SEM-EDS). El análisis de los resultados permitió identificar una aleación de oro/plata/cobre y la presencia de oro de gran pureza, entre 22 y 23 quilates.

Palabras clave: pan de oro, retablos barrocos, microscopía óptica, SEM-EDS.

Abstract: The work that has been developed, aims to provide a new approach to Portuguese Baroque altarpieces, by investigating the gold leaf and its elemental characterization. The qualitative and semiquantitative analysis of the samples collected from the main altarpieces of São Bento da Vitória, Santa Clara and São Francisco churches were performed by optical microscopy (OM) of cross-sections using reflected and polarised light and by scanning electron microscopy (SEM) in combination with energy dispersive spectroscopy (EDS). The results provided a new insight into the gilding technique and on the gold leaf composition, namely the presence of a gold/silver/copper alloy and a very pure gold with 22 to 23 gold carat.

Keywords: gold leaf, wood carved baroque altarpieces, optical microscopy, SEM-EDS

\section{Introdução}

O Norte de Portugal assiste, a partir de meados do século XVII e ao longo do século XVIII, ao desenvolvimento de particularidades específicas ao nível do panorama artístico nacional. Apesar de situado na periferia do discurso artístico europeu, a produção nacional tenta expressar o discurso pós-tridentino de acordo com os critérios estéticos vigentes na Europa, introduzindo no reinado de D. João V (1706-1750) o chamado "gosto moderno" do barroco romano (Ferreira-Alves, 2003, 1989).

No último quartel do século XVII a talha portuguesa sofreu uma profunda transformação, produto de uma longa transição durante a qual surgiram em Portugal várias tendências na ornamentação, 
separando-a da produção espanhola. Segundo Robert Smith a revolução efectuou-se pela acção de dois elementos indispensáveis - a coluna de fuste em espiral, chamado "salomónico" e o remate de arcos concêntricos, que, combinados, deram ao retábulo português uma nova estrutura, mais escultural do que arquitectónica, dinâmica em vez de estática, emprestando-lhe sentido de movimento e efeito de unidade e juntos com folhas de acanto em alto-relevo, esses elementos produriram a primeira manifestação inteiramente barroca da arte portuguesa (Smith, 1962). A este novo estilo Robert Smith denomina-o de Estilo Nacional. O estudo mais recente da talha dourada nortenha enquadra temporalmente o Estilo Nacional a partir da década de 80 de seiscentos até aos anos 20 de setecentos; após a construção do retábulo mor da Sé do Porto, entre 1727 e 1729, tem início o chamado Barroco Joanino, inspirado no barroco romano, que se prolonga até final da década de 50 (Ferreira-Alves, 2003, 1989). É característico deste período um vocabulário decorativo onde predominam conchas, feixes de plumas, palmas, volutas entrelaçadas, grinaldas e festões de flores. Figuram ainda uma diversidade de baldaquinos e sanefas, cortinas e panos, fragmentos de arcos e outros motivos arquitectónicos (Smith, 1962). No interior das igrejas a talha dourada é a manifestação artística mais relevante, conferindo imponência e fausto aos retábulos, surgindo frequentemente associada a outras artes decorativas como o azulejo, a pintura, a escultura e a pintura decorativa, impondo uma nova dimensão a espaços sem relevante expressão arquitectónica. A amplitude atingida por esta conjugação de expressões resulta, muitas vezes, em estruturas de grande complexidade, tanto iconográfica como artística cujo brilho dourado dá especial relevância. A extensa utilização de folha de ouro nos programas decorativos retabulares portugueses barrocos, especialmente nos compreendidos entre o final do séc XVII e séc. XVIII resultou da abundância deste metal precioso proveniente das terras de além-mar, especialmente após a descoberta do complexo mineiro de Minas Gerais, Brasil, no séc. XVII (Guerra, Gondonneau, Barrandon, 1998).

Neste contexto e no sentido de caracterizar a folha de ouro e a técnica de aplicação em retábulos portugueses, foram seleccionados os retábulos-mor pertencentes às Igrejas de São Bento da Vitória (SB), São Francisco (SF) e Santa Clara (SC), considerados dos mais representativos exemplares do Estilo Nacional - São Bento da Vitória - e do Barroco Joanino - São Francisco e Santa Clara.

Sendo escassos os elementos sobre o fornecimento de ouro, a análise dos contratos de douramento existentes e as relações entre mestres douradores e bate-folhas fornecem pistas sobre a dinâmica de circulação e aquisição de ouro e sobre as condições do trabalho a realizar. A análise de diversos contratos deste período reflecte a importância de alguns fornecedores de "pães de ouro", cuja reputação se devia à qualidade da matéria-prima com que trabalhavam e à forma como o ouro era laminado de acordo com a especificação do mestre dourador (Ferreira-Alves, 1989). O contrato de 20 de Outubro de 1755, referente ao conjunto de talha da igreja do Mosteiro de São Bento da Vitória, nomeia o bate-folha Domingos Francisco da Costa como fiador do mestre dourador Manuel Homem Soares. Em 13 de Setembro de 1667 lê-se que as freiras do Convento de Santa Clara estipulam com precisão as condições em que o mestre Manuel de Sousa Sampaio devia efectuar a pintura e douramento utilizando expressões como "ouro bornido" e "ouro lizo". A 2 de Agosto de 1712 os bate-folhas António Carvalho e Baltasar Correia Basto obrigam-se a "bater todo o ouro que necessário lhe for para a obra" de douramento da capela-mor da Ordem Terceira de São Francisco, para o mestre dourador Manuel Pinto Moreiro (Brandão, 1984). O ouro podia ser entregue pelo contratante - fornecido puro com os quilates da Casa da Moeda - ou fornecido pelos bate-folhas, com a exigência de boa qualidade, idêntico à amostra que o cliente havia aprovado e que retinha em seu poder até à conclusão da obra de forma a comprovar a qualidade da folha que os mestres bate-folhas iam entregando (Ferreira-Alves, 1989). Oscilando entre 20 e 24 quilates o ouro apresentava uma cor dourada intensa, sendo designado ao longo dos séculos XVII e XVIII como "ouro subido", a sua venda fazia-se por milheiros, sendo cada milheiro formado por dez livros, cada um com cem pães de ouro (Ferreira-Alves, 1989; Martínez, 1997). A análise dos vários contratos dos séculos XVII e XVIII permite ainda concluir que a técnica utilizada para o douramento, desde a aplicação do aparelho até à aplicação da folha de ouro, seguia o processo tradicional sem grandes variações, sendo algumas receitas muito semelhantes ao que Filipe Nunes (1615) aconselhava no seu tratado a Arte da Pintura. Symmetria e perspectiva. Uma obra aparelhada com todo o rigor pressupunha a aplicação de, pelo menos, três mãos de gesso grosso, três de gesso mate 
e quatro de bolo arménio, sendo o ideal cinco camadas de cada, num total de quinze mãos (Ferreira-Alves, 1989). O contrato de douramento da capela-mor de São Francisco (1712) especifica o aparelhamento com quatro mãos de gesso grosso e cinco de bólus, todas muito finas (Brandão, 1984). A qualidade do aparelho dependia de dois factores: da cola utilizada e do número de mãos de gesso e bólus aplicados, pelo que se tratava de uma etapa crucial do processo de douramento, tanto ao nível técnico, como de tempo de trabalho e montante dispendido na aquisição de material. Depois de aparelhado o suporte dava-se inicio ao douramento, podendo ser efectuado a mordente ou a água, técnicas conhecidas nos séculos XVII e XVIII, sendo o último método mais dispendioso, moroso e de difícil execução. O douramento a água permitia a obtenção de uma superfície polida - "ouro bornido" -, sendo o processo sistematicamente exigido na maior parte dos contratos do século XVII e XVIII, facto que se verifica nos três retábulos estudados.

\section{Metodologia analítica}

As amostras de folha de ouro foram retiradas de zonas discretas, mas representativas, nos diferentes retábulos - São Bento da Vitória, São Francisco e Santa Clara -, nomeadamente a parte de trás das colunas, os painéis fundeiros ou os degraus do trono. Seleccionaram-se locais de difícil acesso de forma a conseguir amostras com reduzida contaminação e pouco desgaste. As amostras foram analisadas quanto à estratigrafia do douramento e à superfície da folha de ouro. As amostras para análise estratigráfica foram englobadas em resina poliéster (BYLAPOX 3085 A e B (2:1)) e polidas numa lixa circular Struers Planopol-V; a observação e registo das amostras por microscopia óptica (OM) foi efectuada com equipamento Zeiss Stemi 2000-C, sob luz polarizada e transmitida com um sistema de luz artificial Zeiss KL 1500 LCD, aquisição de imagem com câmara digital AxioCam MRcS e software de aquisição e tratamento Axio Vs 40 V4.4 Carl Zeiss Vision GmbH. Utilizou-se uma ampliação de 100x. A montagem e observação das amostras seguiu o procedimento aplicado à análise de pintura e escultura (Khandekar, 2003). O estudo da estratigrafia das amostras permite a identificação do número, espessura, adesão e coesão das camadas, a análise da dimensão e forma das partículas (Calvo, 2003), a presença de mais do que um douramento e o estudo das camadas de preparação e bólus. A microscopia electrónica de varrimento (SEM) realizou-se em equipamento Hitachi SU-70 equipado com espectrómetro de energia dispersiva (EDS) Bruker AXS com sistema de nitrogénio livre Quantax 400 EDS e XFlash Silicon Drift Detector (SDD). Aplicou-se uma voltagem de aceleração de $15 \mathrm{kV}$ e uma intensidade de $32 \mathrm{~mA}$. A análise química por EDS foi efectuada em áreas de $200 \mu \mathrm{m}^{2}$, seleccionadas de acordo com a sua homogeneidade e com tempos de aquisição de espectro de 60 segundos. A preparação das amostras foi feita por deposição de carbono.

A combinação de SEM com EDS aumenta a sensibilidade para os elementos mais leves e a resolução espacial é superior para análise por pontos, permitindo ainda a análise da microestrutura por linhas de varrimento e o mapeamento 2D da concentração elementar (Hein and Degrigny, 2008; Guerra, 2004). O mapeamento foi efectuado sob as mesmas condições de obtenção dos espectros EDS, sem eliminação de background. Foram mapeadas áreas de $200 \mu \mathrm{m}^{2}$, com uma ampliação de 8000x e os resultados obtidos são a média de três medições. As áreas mapeadas foram seleccionadas tendo em conta a sua homogeneidade e inexistência de vazios. Os resultados semiquantitativos foram normalizados a $100 \%$.

\section{Resultados e discussão}

O estudo por OM e SEM das amostras provenientes dos retábulos-mor das igrejas de São Bento, Santa Clara e São Francisco revelou, em todas as amostras, a presença de um douramento tradicional, sendo visíveis as camadas de preparação branca, bólus (de cor vermelha) e folha de ouro (Martínez, 1997; Serck-Deawide et al., 2004), não sendo possível identificar o número de aplicações presente em cada uma das camadas [figura 1]. 


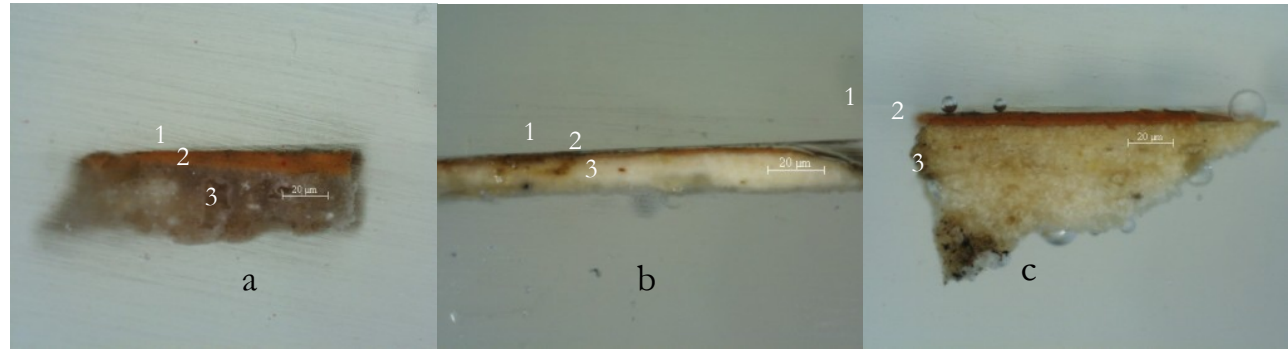

Figura 1. Imagem por OM - luz reflectida. Estratigrafia do douramento das amostras SB (a), SC (b) e SF (c). 1 - Folha de ouro; 2 - Bólus; 3 - Preparação branca.

A análise da preparação branca por EDS, revelou a presença comum de enxofre, cálcio e silício, tendo ainda sido identificado, nas amostras provenientes de Santa Clara e São Francisco, a presença de alumínio. As maiores percentagens de alumínio e silício registaram-se nas amostras de São Francisco [tabela 1]. O estudo elementar da folha de ouro por EDS permitiu identificar a presença de uma liga de ouro/prata/cobre com uma percentagem de ouro muito elevada, variando entre os 22 e os 23 quilates (os resultados foram normalizados a 100\%) [tabela 2].

Tabela 1. Percentagem em peso (wt/\%) dos elementos identificados na preparação branca.

\begin{tabular}{|l|l|l|l|l|l|}
\cline { 2 - 6 } \multicolumn{1}{c|}{ s } & \multicolumn{4}{l|}{ Elementos (wt/\%) } \\
\hline Amostras & Ca & S & Si & Al & O \\
\hline SB & 28,21 & 20,26 & 0,29 & ------ & 51,24 \\
\hline SC & 28,54 & 19,92 & 0,15 & 0,19 & 51,20 \\
\hline SF & 23,71 & 20,19 & 1,89 & 1,89 & 52,33 \\
\hline
\end{tabular}

Tabela 2. Percentagens relativas da composição da liga de ouro e quilates.

\begin{tabular}{|l|l|l|l|l|}
\cline { 2 - 5 } \multicolumn{1}{c|}{} & \multicolumn{2}{l|}{$\%$} \\
\hline Amostras & $\mathrm{Au}$ & $\mathrm{Ag}$ & $\mathrm{Cu}$ & Quilates \\
\hline SB & 91,99 & 2,9 & 5,11 & 22,08 \\
\hline SC & 94,91 & 0,91 & 4,18 & 22,78 \\
\hline SF & 91,65 & 4,41 & 3,94 & 22,00 \\
\hline
\end{tabular}


As amostras revelaram a presença comum de diversos elementos, contributo das camadas de preparação e bólus e da folha de ouro [figura 2]. O mapeamento da superfície forneceu a distribuição topográfica dos elementos presentes nas amostras e a sua concentração, correspondendo as áreas mais intensas às maiores concentrações e as mais esbatidas a uma menor concentração dos elementos (Goldstein, J. I., 1975). É visível a presença de ouro, prata e cobre pertencentes à folha de ouro, e alumínio e silício contribuições do bólus, uma camada argilosa rica em alumino-silicatos [figura 3].

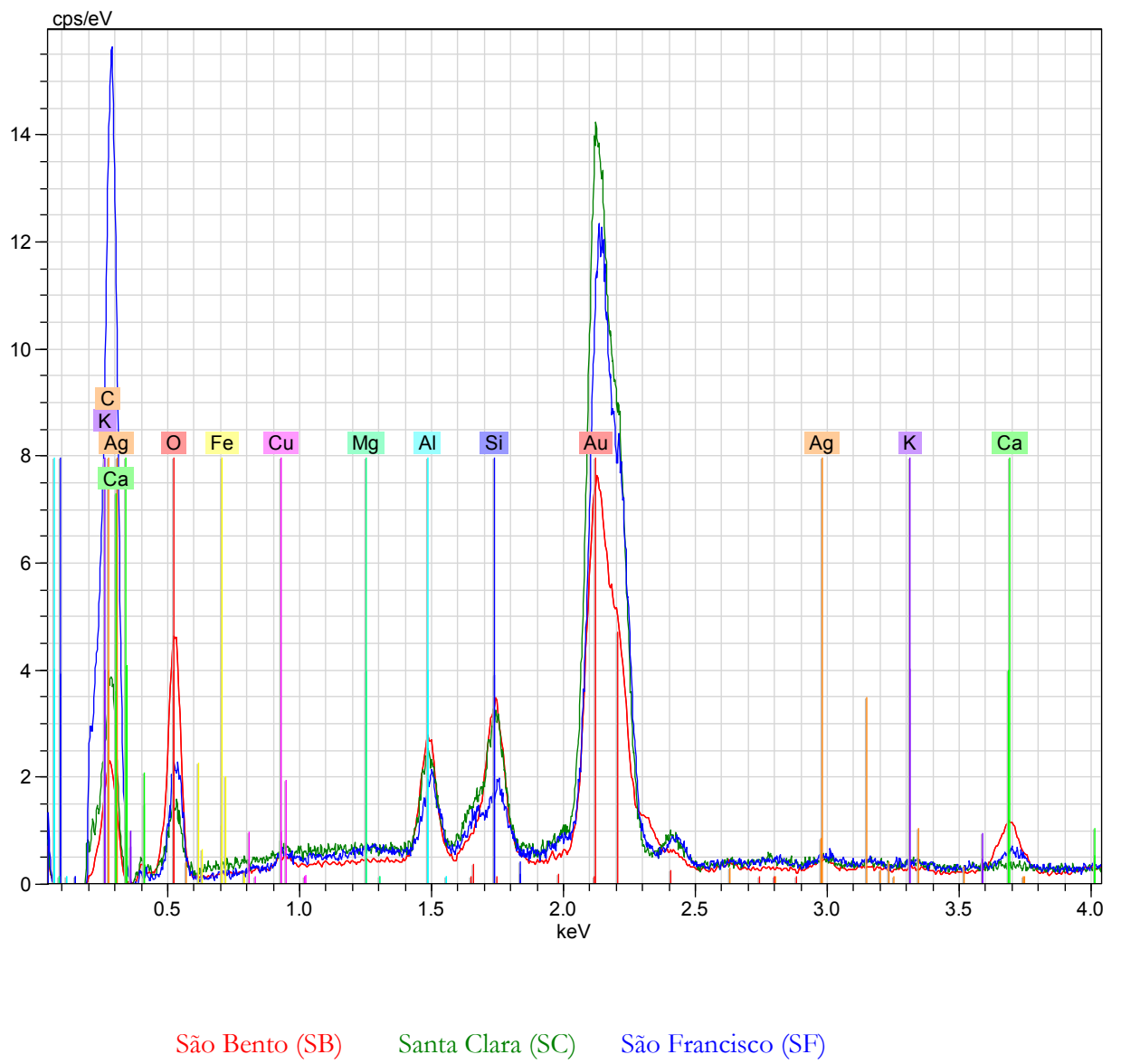

Figura 2. Espectro EDS dos elementos maioritários presentes na folha de ouro - valores médios. Amostras SB, SC e SF.

A análise por SEM-EDS permite a determinação dos elementos maioritários na liga de ouro bem como a definição das percentagens relativas dos elementos nas diferentes camadas que constituem um douramento tradicional. A análise de elementos traço na folha de ouro, tais como o paládio, telúrio, ródio, crómio ou ruténio, não é possível através desta técnica devido aos limites de detecção do EDS, à sobreposição de picos e à interferência de ruídos de fundo (bremsstrablung background) (Bidarra, Coroado, Rocha, 2009). De forma a poder proceder-se à sua identificação e determinação serão necessários exames complementares tais como a análise por espectrometria de massa acoplada a plasma indutivo (ICP-MS) e utilização de radiação sincrotrão (SR). 

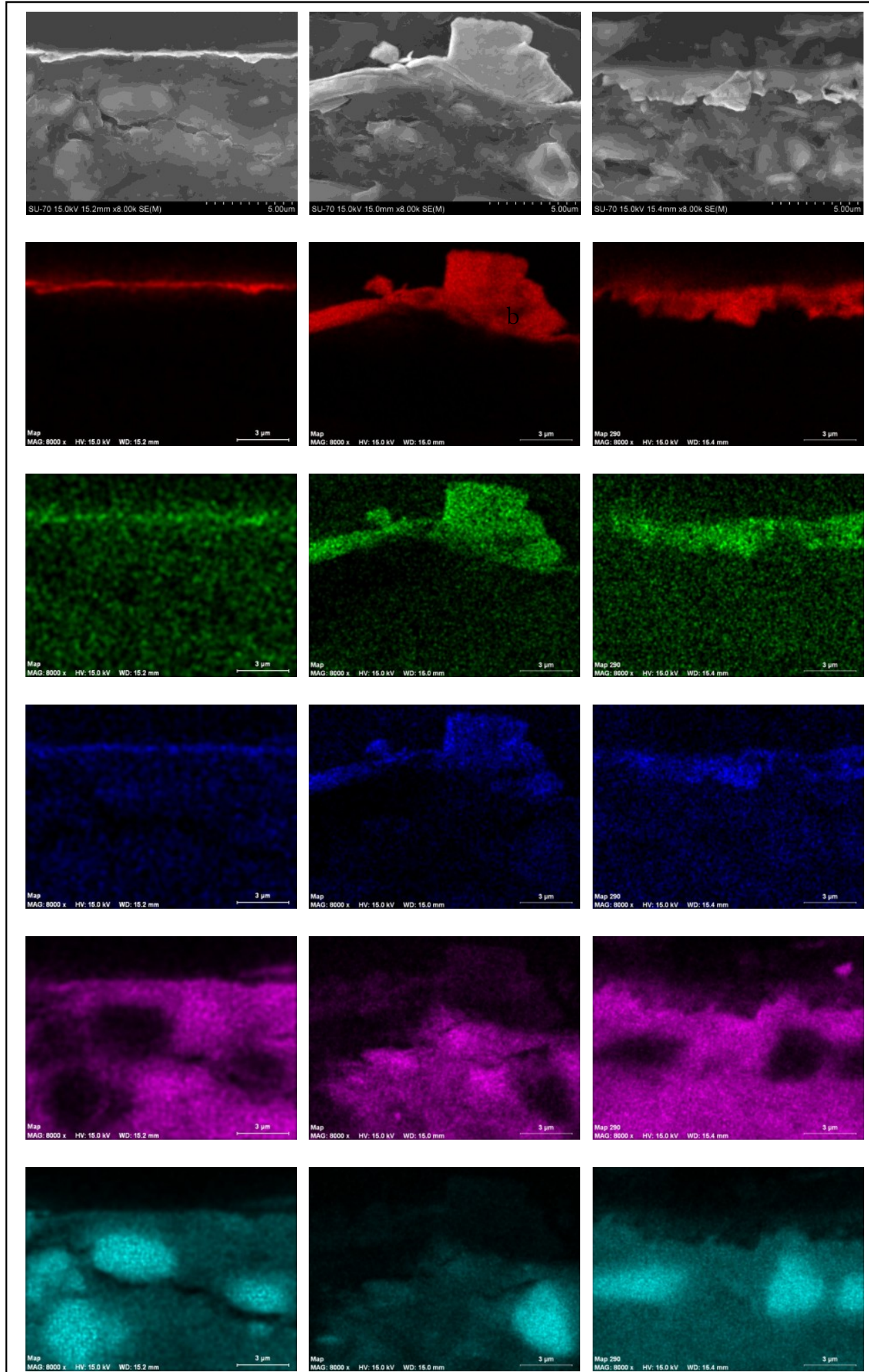

Figura 3. Imagem SEM e mapeamento da superfície (8000x). a) SB; b) SC e c) SF. Au / Ag / Cu / Si / Al. 


\section{Conclusões e perspectivas de desenvolvimento}

A determinação de elementos maioritários da folha de ouro através de SEM-EDS permitiu identificar a presença de ouro, prata e cobre em todas amostras analisadas, sendo clara a contribuição da folha de ouro para a presença destes elementos; determinou-se ainda a presença de alumínio e silício (contribuição da camada de bólus) e enxofre e cálcio (camada de preparação). A análise por SEM-EDS de elementos traço e minoritários, não apresentou resultados conclusivos, encontrando-se em curso uma segunda fase experimental de análise das amostras por espectrometria de massa (ICP-MS) e radiação de sincrotrão (SR). A observação por OM continua a ser um contributo valioso na análise da estratigrafia de um douramento, fornecendo dados importantes quanto ao número, cor e espessura das camadas, tamanho das partículas e presença de mais do que um douramento.

Confirmou-se a presença de "ouro subido", de elevada pureza - entre 22 e 22 3/4 quilates, bem como a presença de um douramento tradicional, tal como especificado na generalidade dos contratos dos séculos XVII e XVIII.

Os resultados obtidos fazem parte de um estudo mais amplo de análise composicional do ouro, de tipificação do seu envelhecimento e de estabelecimento de relações de proveniência (extracção), que irão permitir uma melhor abordagem à conservação e restauro da folha de ouro. Pretende-se verificar a possibilidade de estabelecer uma identidade distinta para o ouro utilizado nos diferentes retábulos, estabelecendo uma diferente abordagem ao estudo destas obras através da análise composicional da folha de ouro.

\section{Agradecimentos}

Fundação Portuguesa para a Ciência e a Tecnologia (FCT) - FRH/BD/38593/2007.

GeoBioTec Research Centre - Universidade de Aveiro

Centro Tecnológico da Cerâmica e do Vidro (CTCV) - Coimbra.

\section{Bibliografía}

(no prelo) Bidarra, A., Coroado, J., Rocha, F. (2009), "Gold leaf analysis of three Porto baroque altarpieces", Archeo Science, Revue d'Archéométrie.

BRANDÃO, D. de P. (1984). Obra de talha dourada, ensamblagem e pintura na cidade e diocese do Porto. Documentação I, SÉCULOS XV A XVII, S/E, PORTO.

CALVO, A. (2003). Conservación y Restauración - Materiales técnicas y procedimientos - De la A a la Z. Tercera EDICIÓN. EDICIONES DEL SERBAL, BARCELONA.

FERrEIRA-ALVES, N. M. (1989). A Arte da Talha no Porto na Época Barroca. Artistas e clientela. Materiais e técnica. Vol.I e II. Arquivo Histórico, Câmara Municipal do Porto.

FERREIRA-ALVES, N. M. (2004). O douramento e a policromia no Norte de Portugal à luz da documentação dos séculos XVII e XVII. Policromia - A escultura policromada religiosa dos séculos XVII e XVIII. Estudo comparativo das técnicas, alterações e conservação em Portugal, Espanha e Bélgica. Actas do Congresso Internacional, Lisboa 29, 30 e 31 de Outubro de 2002. Instituto Português de Conservação e Restauro, Lisboa, 17-22.

GOLDSTEIN, J. I. (1975). Electron beam - specimen interaction. In J. I. Goldstein and H. Yakowitz (dir.). Practical scanning electron microscopy. Electron and ion microprobe analysis. Plenum Press, New York, 49 - 94. 
GUERRA, M. F., Calligaro, T. (2004). Gold traces to trace gold. Journal of Archaeological Science, 31: 1199-1208.

GUERRA, M. F. (2004). Fingerprinting ancient gold with proton beams of different energies. Nuclear Instruments and Methods in Physics Research, Serie B, 226: 185-198.

GUERRA, M. F., GONDONNEAU, A., BARRANDON, J.-N. (1998). South American precious metals and the European economy: a scientific adventure in the discoveries time. Nuclear Instruments and Methods in Physics Research, Serie B, 136-138: 875-879.

HEIN, A., DEGRIGNY, C. (2008). The application of non-destructive technologies for the damage assessment of metal objects. In V. Argyropoulos (dir.). Metals and Museums in the Mediterranean, Protecting, Preserving and Interpreting. The PROMET Consortium, Greece, 125-140.

KHANDEKAR, N. (2003). Preparation of cross sections from easel paintings. Reviews in Conservation, 4: 5264.

MARTÍNEZ, E. (1997). Tratado del dorado, plateado y su policromia. Tecnología, conservación y restauración. Departamento de Conservación y Restauración de Bienes Culturales, Universidad Politécnica Valência.

NUNES, F. (1982). Arte da pintura. Symmetria e perspectiva. Editorial Paisagem, Porto (fac-simile da edição de 1615).

SERCK-dEAWIDE, m., rIBEIRO, i., et al. (2004). Les techniques utilisées dans l'art baroque religioux des $\mathrm{XVII}^{\mathrm{e}}$ et XVIII ${ }^{\mathrm{e}}$ siècles au Portugal, en Espagne et en Belgique. In A. I. Seruya (dir.). Policromia - A escultura policromada religiosa dos séculos XVII e XVIII. Estudo comparativo das técnicas, alterações e conservação em Portugal, Espanha e Bélgica. Actas do Congresso Internacional, Lisboa 29, 30 e 31 de Outubro de 2002. Instituto Português de Conservação e Restauro, Lisboa, 119-155.

SMITH, R. (1962). A talha em Portugal. Livros Horizonte, Lisboa.

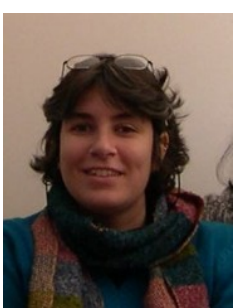

\section{A. Bidarra}

Departamento de Geociências / GeoBio Tec Research Centre - Universidade de Aveiro Campus Universitário de Santiago - 3810-193 Aveiro, Portugal bidarra.ana@gmail.com

Ana Bidarra. Licenciada em Conservação e Restauro pelo Instituto Politécnico de Tomar, Mestre em Geociências pela Universidade de Aveiro, com área de especialização no estudo dos pigmentos brancos estruturados para reintegração de obras de arte. Em doutoramento na Universidade de Aveiro, com o tema de investigação "Estudo tecnológico e composicional da folha de ouro dos retábulos barrocos portugueses". Conservadora-restauradora desde 1999. 


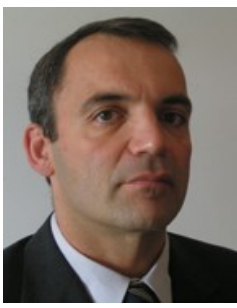

\section{J. Coroado}

Departamento de Arte, Conservação e Restauro, Instituto Politécnico de Tomar Quinta do Contador, Estrada da Serra, PT - 2300-313 Tomar, Portugal, jcoroado@ipt.pt

João Coroado. Professor Coordenador e Director do Departamento de Arte, Conservação e Restauro do Instituto Politécnico de Tomar e membro permanente da Unidade de Investigação GeoBioTec, financiada pela Fundação de Ciência e Tecnologia tendo sido avaliada em 2009 com a classificação de Muito Bom

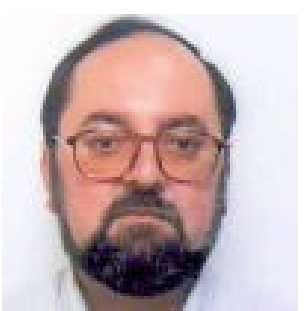

F. Rocha

Departamento de Geociências / GeoBio Tec Research Centre - Universidade de Aveiro

Campus Universitário de Santiago - 3810-193 Aveiro

tavares.rocha@ua.pt

Fernando Rocha. Licenciado em Geologia pela Universidade de Lisboa, Doutorado e Agregado em Geociências pela Universidade de Aveiro, com área de especialização em estudos mineralógicos e geoquímicos de materiais argilosos, aplicados a problemas de recursos minerais, património, sedimentologia, paleoclimatologia, riscos geológicos e impactos ambientais. Professor Catedrático da Universidade de Aveiro desde 2002. Coordenador da Unidade de Investigação "Geobiociências, Geotecnologias e Geo-engenharias Geobiotec" desde a sua fundação em 2007.

Artículo recibido el 31/03/2010.

Artículo aceptado el 27/06/2010 